\title{
Erratum to: Highway traffic accident prediction using VDS big data analysis
}

\author{
Seong-hun Park ${ }^{1}$ • Sung-min Kim ${ }^{1}$. \\ Young-guk $\mathrm{Ha}^{2}$
}

\section{Erratum to: J Supercomput 2016 DOI 10.1007/s11227-016-1624-Z}

The original version of this article unfortunately contained a mistake. The section "Acknowledgment" was missing. The Acknowledgment is given below.

Acknowledgments This work was supported by the ICT R\&D program of MSIP/IITP. [R-20150224000386, A Development of Software Technology for the Generation of Detailed Map Progressively Updated and the Driving Situation Awareness based on Cloud Computing.]

The online version of the original article can be found under doi:10.1007/s11227-016-1624-z.

Young-guk Ha

ygha@konkuk.ac.kr

Seong-hun Park

wolfire@konkuk.ac.kr

Sung-min Kim

allmax75@konkuk.ac.kr

1 Konkuk University, 1007, Newmillenium Hall, Hwayang-dong, Gwangjin-gu, Seoul, Korea

2 Konkuk University, 903, Newmillenium Hall, Hwayang-dong, Gwangjin-gu, Seoul, Korea 